PROCEEDINGS OF THE

AMERICAN MATHEMATICAL SOCIETY

Volume 129, Number 5, Pages 1257-1262

S 0002-9939(00)05749-X

Article electronically published on October 12, 2000

\title{
LES TRANSFORMATIONS DE CREMONA STELLAIRES
}

\author{
IVAN PAN
}

(Communicated by Michael Stillman)

\begin{abstract}
RÉSUMÉ. On construit explicitement toutes les transformations de Cremona de $\mathbf{P}^{n}$ qui satisfont à la propriété suivante: il existe $P_{1}, P_{2} \in \mathbf{P}^{n}$ tels que les droites par $P_{1}$ sont envoyées sur les droites par $P_{2}$. On caractérise de plusieurs manières ces transformations et pour chaque entier non-négatif $d$ on donne des formules pour la dimension de l'ensemble constitué de celles qui ont degré $d$.

Abstract. We construct the Cremona transformations of $\mathbf{P}^{n}$ satisfying the following property: there exist $P_{1}, P_{2} \in \mathbf{P}^{n}$ such that the image of all straight lines through $P_{1}$ are straight lines through $P_{2}$. We characterise these transformations, and for all non-negative integer $d$ we give a formula for the dimension of the set of those whose degree is $d$.
\end{abstract}

\section{INTRODUCTION}

Tout au long de ce travail, $k$ désignera un corps algébriquement clos de caractéristique zéro, $A:=k\left[x_{0}, \ldots, x_{n}\right]$ l'anneau des polynômes à $(n+1)$ variables sur $k$ et pour $d$ entier non-négatif, $A_{d}$ le sous-espace vectoriel des polynômes homogènes de degré $d$ (bien sûr $A_{0}=k$ et $A_{l}=0$ si $l<0$ ).

Soit $t=\left(t_{1}, \ldots, t_{n}\right) \in\left(k\left[x_{1}, \ldots, x_{n}\right]_{r}\right)^{n}$ avec $r \geq 1$ et $\operatorname{pgcd}\left(t_{1}, \ldots, t_{n}\right)=1$. Pour $d \geq r$ on se donne $q \in A_{d-r}, g \in A_{d}$ avec $\operatorname{pgcd}(q, g)=1$ et on définit

$$
T_{g, q, t}: \mathbf{P}^{n} \mathbf{P}^{n}
$$

par

$$
T_{g, q, t}=\left[g, q t_{1}, \ldots, q t_{n}\right] .
$$

Dans ce travail on montre que $T_{g, q, t}$ est de Cremona si et seulement si

(i) $\bar{T}:=\left[t_{1}, \ldots, t_{n}\right]: \mathbf{P}^{n-1} \rightarrow \mathbf{P}^{n-1}$ est de Cremona, et

(ii) $m_{O}(g) \geq d-1$ et $m_{O}(g)+m_{O}(q) \in\{2 d-r-2,2 d-r-1\}$,

où $m_{O}(f)$ indique l'ordre d'annulation de $f$ en $O$; pour $n=2$ le cas où $t=i d$ correspond aux transformations de de Jonquières (voir [7] et [4]). On caractérise de plusieurs manières ces transformations et en particulier on montre que l'ensemble des transformations $T_{g, q, t}$ est, à changements de variables à la source et au but près, l'ensemble des transformations de Cremona pour lesquelles il existe $P_{1}, P_{2} \in \mathbf{P}^{n}$ tels que l'espace des droites par $P_{1}$ s'envoient birationnellement sur l'espace des droites

Received by the editors July 20, 1999.

1991 Mathematics Subject Classification. Primary 14E07, 14N99.

Key words and phrases. Transformation de Cremona, stellaire.

Au moment de la redaction de ce travail, l'auteur était attaché à l'Instituto de MatemáticaUFRGS en qualité de boursier du CNPq-Brésil. 
par $P_{2}$; on appele stellaires ces dernières. Finalement, on donne des formules pour la dimension de l'ensemble des transformations stellaires de degré $d$.

J'aimerais remercier Thierry Vust pour son aide dans la redaction de ce travail, sans laquelle elle ne se serait jamais achevée.

\section{LES TRANSFORMATIONS STELLAIRES}

Définition 2.1. Soit $F: \mathbf{P}^{n} \rightarrow \mathbf{P}^{n}$ une transformation de Cremona. On dit que $F$ est stellaire s'il existe $P_{1}, P_{2} \in \mathbf{P}^{n}$ et $\bar{F}: \mathbf{P}\left(k^{n+1} / P_{1}\right)_{--\rightarrow} \mathbf{P}\left(k^{n+1} / P_{2}\right)$ birationnelle tels que

$$
\bar{F} \circ \pi_{1}=\pi_{2} \circ F
$$

où $\pi_{i}: \mathbf{P}\left(k^{n+1}\right)=\mathbf{P}_{--\rightarrow}^{n} \mathbf{P}\left(k^{n+1} / P_{i}\right)$ est la projection de centre $P_{i}$.

Introduisant l'étoile de $P_{i}$, à savoir la sous-variété $E t\left(P_{i}\right)$ de la grassmannienne des droites de $\mathbf{P}^{n}$ constituée des droites passant par $P_{i}$, on voit que $F$ est stellaire si et seulement si $F$ induit une application birationnelle $E t(F): E t\left(P_{1}\right)_{--\rightarrow E t}\left(P_{2}\right)$.

On considère l'éclatement $\sigma_{i}: B l_{P_{i}}\left(\mathbf{P}^{n}\right)_{--\rightarrow} \mathbf{P}^{n}$ de $P_{i}$ dans $\mathbf{P}^{n} ;$ alors $\rho_{i}:=$ $\pi_{i} \circ \sigma_{i}$ est un morphisme qui presente $B l_{P_{i}}\left(\mathbf{P}^{n}\right)$ comme fibré en droites projectives sur $\mathbf{P}^{n-1}$ : c'est $\mathbf{P}\left(\mathcal{O}_{\mathbf{P}^{n-1}}(-1) \oplus \mathcal{O}_{\mathbf{P}^{n-1}}\right)$. On note $\hat{F}: B l_{P_{1}}\left(\mathbf{P}^{n}\right)_{--\rightarrow B} B l_{P_{2}}\left(\mathbf{P}^{n}\right)$ l'application birationnelle telle que $\sigma_{2} \circ \hat{F}=F \circ \sigma_{1}$. Alors $F$ est stellaire si et seulement s'il existe une application birationnelle $\bar{F}: \mathbf{P}\left(k^{n+1} / P_{1}\right)_{--\rightarrow} \mathbf{P}\left(k^{n+1} / P_{2}\right)$ telle que

$$
\rho_{2} \circ \hat{F}=\bar{F} \circ \rho_{1} \text {. }
$$

Un couple $\left(P_{1}, P_{2}\right)$ comme dans la définition ci-dessus s'appelle un centre pour la transformation stellaire $F ;\left(P_{2}, P_{1}\right)$ est un centre pour $F^{-1}$. On dira aussi que $P_{1}$ est un sommet pour $F$ et $P_{2}$ un sommet pour $F^{-1}$.

Exemple 2.1. Un automorphisme $F$ de $\mathbf{P}^{n}$ est une transformation stellaire de centre $(P, F(P))$ pour tout $P \in \mathbf{P}^{n}$.

Exemple 2.2. La transformation de Cremona de $\mathbf{P}^{n}$

$$
F_{n}=\left[x_{1} \cdots x_{n}, x_{0} x_{2} \cdots x_{n}, \ldots, x_{0} \cdots x_{n-1}\right]=\left[\frac{1}{x_{0}}, \ldots, \frac{1}{x_{n}}\right]
$$

est stellaire de centre $\left(O_{i}, O_{i}\right), i=0,1, \ldots, n$, où $O_{i}:=[0, \ldots, 1, \ldots, 0]$.

On note $\mathbf{S t}\left(\mathbf{P}^{n}\right)$ (resp. $\mathbf{S t}_{O}\left(\mathbf{P}^{n}\right)$ ) l'ensemble des transformations stellaires de $\mathbf{P}^{n}$ (resp. de centre $(O, O)$ où $O=[1,0, \ldots, 0])$. Le groupe $P G L(n+1) \times P G L(n+1)$ opère dans $\mathbf{S t}\left(\mathbf{P}^{n}\right)$ par composition à la source et au but; de plus

$$
\mathbf{S t}\left(\mathbf{P}^{n}\right)=(P G L(n+1) \times P G L(n+1)) \cdot \mathbf{S t}_{O}\left(\mathbf{P}^{n}\right)
$$

et $\mathbf{S t}_{O}\left(\mathbf{P}^{n}\right)$ est un sous-groupe du groupe de Cremona $\operatorname{Cr}\left(\mathbf{P}^{n}\right)$ de $\mathbf{P}^{n}$.

Proposition 2.1. L'application $F \longrightarrow \bar{F}$ induit une suite exacte scindée

$$
1 \rightarrow P G L\left(2, k\left(y_{1}, \ldots, y_{n-1}\right)\right) \hookrightarrow \mathbf{S t}_{O}\left(\mathbf{P}^{n}\right) \longrightarrow \mathbf{C r}\left(\mathbf{P}^{n-1}\right) \rightarrow 1
$$

Preuve. On a la version "locale" suivante: l'application $F: k^{n}-\rightarrow k^{n}$ est stellaire de centre $(O, O)$ si et seulement s'il existe $\bar{F}: k^{n-1}-\rightarrow k^{n-1}$ birationnelle tel que le diagramme

$$
\begin{array}{rcl}
k^{n-1} \times k & -F & k^{n-1} \times k \\
\pi \downarrow & & \downarrow \pi \\
k^{n-1} & -\bar{F} & k^{n-1}
\end{array}
$$


commute. Il s'ensuit aussitot que la suite de l'enoncé est exacte; elle est scindée par

$$
\bar{F} \longmapsto[(y, z) \mapsto(\bar{F}(y), z)], y \in k^{n-1}, z \in k .
$$

Voici encore une déscription géométrique des transformations stellaires.

Soit $\bar{T}: \mathbf{P}^{n-1}{ }_{--\rightarrow} \mathbf{P}^{n-1}$ et $T: \mathbf{P}_{--\rightarrow} \mathbf{P}^{n}$ deux applications rationnelles telles que $\pi \circ T=\bar{T} \circ \pi$ où $\pi: \mathbf{P}^{n}{ }_{--} \mathbf{P}^{n-1}$ est la projection de centre $O$

$$
\left[x_{0}, x_{1}, \ldots, x_{n}\right] \longmapsto\left[x_{1}, \ldots, x_{n}\right] .
$$

Si $\bar{T}=\left[t_{1}, \ldots, t_{n}\right]$ où $t_{i} \in k\left[x_{1}, \ldots, x_{n}\right]_{r}$ avec $\operatorname{pgcd}\left(t_{1}, \ldots, t_{n}\right)=1$, il existe $g \in$ $A_{d}, q \in A_{d-r}$ avec $\operatorname{pgcd}(q, g)=1$, qui sont uniques à multiplication par un scalaire non-nul près, tels que

$$
T_{g, q, t}:=T=\left[g, q t_{1}, \ldots, q t_{n}\right] .
$$

Remarque 2.1. Dans la preuve du résultat principal de [6] on montre que si $n>2$, tout ensemble de générateurs de $\mathbf{C r}\left(\mathbf{P}^{n}\right)$ doit contenir une famille non dénombrable d'éléments de $\mathbf{S t}_{O}\left(\mathbf{P}^{n}\right) \backslash P G L(n+1)$.

Finalement, pour une application rationnelle $F=\left[f_{0}, \ldots, f_{m}\right]: \mathbf{P}_{--\rightarrow} \mathbf{P}^{m}$, avec $\operatorname{pgcd}\left(f_{0}, \ldots, f_{m}\right)=1$, on note $B(F)$ le schéma de base définit par l'idéal $\left(f_{0}, \ldots, f_{m}\right)$ et $\Lambda_{F}$ le sous-système linéaire engendré par les $f_{i}$ : celui-ci est l'ensemble des hypersurfaces d'équations $\sum_{i=0}^{m} a_{i} f_{i}=0,\left[a_{0}, \ldots, a_{m}\right] \in \mathbf{P}^{m}$. Si $P \in \mathbf{P}^{n}$, la multiplicité de $\Lambda_{F}$ en $P$ est le nombre entier non-négatif

$$
m_{P}\left(\Lambda_{F}\right):=\min \left\{m_{P}(S): S \in \Lambda_{F}\right\},
$$

où $m_{P}(S)$ est la multiplicité de l'hypersurface $S$ en $P$; si $S=\{f=0\}$, ce nombre est l'ordre d'annulation $m_{P}(f)$ de $f$ en $P$.

Proposition 2.2. Les assertions suivantes sont équivalentes:

(1) $T_{g, q, t}$ est birationnelle;

(2) $\bar{T}$ est birationnelle et $m_{O}\left(\Lambda_{T_{g, q, t}}\right)=d-1$;

(3) $\bar{T}$ est birationnelle, $m_{O}(g) \geq d-1$ et $m_{O}(g)+m_{O}(q) \in\{2 d-r-2,2 d-r-1\}$.

En particulier, si $n>2$ et $d>1$ le point $O$ est un point singulier de $B\left(T_{g, q, t}\right)$.

Preuve. Notons $\Lambda:=\Lambda_{T_{g, q, t}}$.

$(1 \Longleftrightarrow 2)$ Au dehors de $\{g=q=0\} \cup\left\{g=t_{1}=\cdots=t_{n}=0\right\}$, l'intersection de $n$ éléments génériques dans $\Lambda$ est l'intersection d'une hypersurface générique dans $\Lambda$ avec l'intersection de $n-1$ hypersurfaces dans $\mathbf{P}^{n}$ de la forme $\sum_{i=1}^{i=n} \lambda_{i} t_{i}=0$ en dehors de $\left\{t_{1}=\cdots=t_{n}=0\right\}$; d'où l'assertion.

$(2 \Longleftrightarrow 3)$ Observons d'abord que $m_{O}\left(t_{i}\right)=r$ pour tout $i$. Par définition $m_{O}(\Lambda)=d-1$ si et seulement si $m_{O}(g), m_{O}\left(q t_{i}\right) \geq d-1$ et l'un des $g, q t_{1}, \ldots q t_{n}$ a multiplicité $d-1$ en $O$. L'assertion suit du fait que $m_{O}\left(q t_{i}\right)=m_{O}(q)+m_{O}\left(t_{i}\right)$.

Montrons maintenant la dernière assertion. D'une part si $r=1$ on montre sans peine que l'idéal $\left(x_{1}, \ldots, x_{n}\right)$ définit un point immergé de $B\left(T_{g, q, t}\right)$ : c'est l'annulateur de $q$ (ici on a utilisé $n>2$ ); d'autre part, si $r>1$ on constate que la matrice jacobienne associée à l'idéal $\left(g, q t_{1}, \ldots, q t_{n}\right)$ a rang $\leq 1$ en $O$. On en déduit l'assertion.

Le degré commun des $f_{i}$ dans l'écriture de $F=\left[f_{0}, \ldots, f_{m}\right]$ s'appelle le degré de $F$, qu'on note $\operatorname{deg}(F)$. 
Remarque 2.2. Parmi les hypersurfaces de degré $d>2$ qui possèdent au moins un point de multiplicité $d-1$, celles qui n'en possèdent qu'un seul constituent un sousensemble dense. On peut donc dire qu'une transformation stellaire "générique" de degré $>2$ ne possède qu'un seul sommet.

Exemple 2.3. Les transformations de Cremona à schéma de base lisse et non discret (pour l'existence voir [5] et [1]) ne sont pas stellaires.

Exemple 2.4. Soient $d \geq 1$ et $n \geq 2$. En prenant $r=1$, on construit des exemples de transformation stellaires de $\mathbf{P}^{n}$ de degré $d$.

Le corollaire suivant donne un critère plus calculatoire pour décider sur la birationnalité d'un candidat à transformation stellaire. Pour $f \in A_{d}$ on pose $f=$ $x_{0}^{d} f_{0}+\cdots+x_{0} f_{d-1}+f_{d}$ où $f_{i} \in k\left[x_{1} \ldots, x_{n}\right]$.

Corollaire 2.3. On a les assertions équivalentes suivantes

(1) $T_{g, q, t}$ est birationnelle;

(2) $\bar{T}$ est birationnelle, $g=x_{0} g_{d-1}+g_{d}, q=x_{0} q_{d-r-1}+q_{d-r}$ et $g_{d} q_{d-r-1}-$ $g_{d-1} q_{d-r} \neq 0$.

Preuve. Par la proposition, il suffit de démontrer que les assertions suivantes sont équivalentes

(1) $g_{d-1} \neq 0$ ou $q_{d-r-1} \neq 0$;

(2) $g_{d} q_{d-r-1}-g_{d-1} q_{d-r} \neq 0$.

On montre $(1 \Rightarrow 2)$, car l'autre assertion est évidente.

Supposons par l'absurde

$$
g_{d} q_{d-r-1}=g_{d-1} q_{d-r} .
$$

Puisque $q_{d-r-1}=g_{d-1}=0$ n'est pas possible et $q \neq 0$, on a $q_{d-r-1} \neq 0$; soit

$$
q_{d-r-1}=h h^{\prime}
$$

où $h\left|g_{d-1}, h^{\prime}\right| q_{d-r}$ (éventuellement $h=1$ ou $h^{\prime}=1$ ). Posons

$$
g_{d-1}=\bar{g} h, q_{d-r}=\bar{q} h^{\prime} .
$$

De l'équation (2) suit

$$
g_{d}=\bar{g} \bar{q} .
$$

Donc

$$
g=\bar{g}\left(x_{0} h+\bar{q}\right), q=h^{\prime}\left(x_{0} h+\bar{q}\right),
$$

ce qui contredit $\operatorname{pgdc}(g, q)=1$ et démontre l'assertion.

\section{Quelques CAlCUls De Dimension}

On fixe $n \geq 1$. Soient $r \leq d$ et $\mathcal{E} \subset \mathbf{C r}\left(\mathbf{P}^{n-1}\right)$ un sous-ensemble constructible constitué de transformations de degré $r$ (i.e. $\left.\mathcal{E} \subset \mathbf{P}\left(k\left[x_{1}, \ldots, x_{n}\right]_{r}^{n}\right)\right)$. On note

$$
\begin{aligned}
\mathbf{S t}_{O}(\mathcal{E})_{d} & :=\left\{F \in \mathbf{S t}_{O}\left(\mathbf{P}^{n}\right): \operatorname{deg}(F)=d, \bar{F} \in \mathcal{E}\right\}, \\
\mathbf{S t}(\mathcal{E})_{d} & :=(P G L(n+1) \times P G L(n+1)) \cdot \mathbf{S t}_{O}(\mathcal{E})_{d} ;
\end{aligned}
$$

observer que dans le cas où $r=1$ et $\emptyset \neq \mathcal{E} \subset P G L(n)$ on a $\mathbf{S t}(\mathcal{E})_{d}=\mathbf{S t}(P G L(n))_{d}$.

Pour $d=1$, on a $r=1$ et alors, $\mathcal{E} \subset P G L(n)$; on voit facilement que si $\mathcal{E} \neq \emptyset$, alors

$$
\mathbf{S t}(\mathcal{E})_{1}=P G L(n+1) .
$$

On traite à continuation le cas où $d \geq 2$. 
Considérons la fonction polynomiale

$$
p: \mathbf{Z} \times \mathbf{Z} \times \mathbf{Z} \longrightarrow \mathbf{Z}
$$

donnée par

$p(x, y, z)=\left(\begin{array}{c}x+y-1 \\ y\end{array}\right)+\left(\begin{array}{c}x+y-2 \\ y-1\end{array}\right)+\left(\begin{array}{c}x+y-z-1 \\ y-z\end{array}\right)+\left(\begin{array}{c}x+y-z-2 \\ y-z-1\end{array}\right)-2$,

où $\mathbf{Z}$ est l'ensemble des nombres entiers. On a

Proposition 3.1. Soit $d>2$. Si $\mathcal{E}$ est irréductible, alors $\mathbf{S t}(\mathcal{E})_{d}$ est un sousensemble constructible et irréductible d'une variété quasi-projective tel que

$$
\operatorname{dim} \operatorname{St}(\mathcal{E})_{d}=\left\{\begin{array}{l}
\operatorname{dim} \mathcal{E}+p(n, d, r)+2 n+1 \quad \text { si } r>1 \\
\operatorname{dim} P G L(n)+p(n, d, r)+2 n+1 \quad \text { si } r=1 .
\end{array}\right.
$$

Preuve. Considérons les sous-ensembles

$$
\begin{gathered}
U_{d, r}^{2}:=\left\{(g, q) \in A_{d} \times A_{d-r}: \operatorname{pgcd}(g, q)=1\right\}, \\
U_{r}^{n}:=\left\{\left(t_{1}, \ldots, t_{n}\right) \in k\left[x_{1}, \ldots, x_{n}\right]_{r}^{n}: \operatorname{pgcd}\left(t_{1}, \ldots, t_{n}\right)=1\right\} .
\end{gathered}
$$

L'application $\left((g, q),\left(t_{1}, \ldots, t_{n}\right)\right) \mapsto\left(g, q t_{1}, \ldots, q t_{n}\right)$ induit un morphisme injectif

$$
\phi: U_{d, r}^{2} \times U_{r}^{n} /\left(k^{*}\right)^{2} \longrightarrow \mathbf{P}\left(k\left[x_{0}, \ldots, x_{n}\right]_{d}^{n}\right)
$$

où $\left(k^{*}\right)^{2}$ opère par $(a, b) \cdot(g, q, t) \mapsto(a b g, a q, b t)$; l'image de $\phi$ contient les éléments de degré $d$ dans $\mathbf{S t}_{O}\left(\mathbf{P}^{n}\right)$. De la proposition 2.2 on déduit que $\mathbf{S t}_{O}(\mathcal{E})_{d}$ est irréductible et

$$
\operatorname{dim} \mathbf{S t}_{O}(\mathcal{E})_{d}=\operatorname{dim} \mathcal{E}+1+p(n, d, r) .
$$

Par ailleurs, observer que $\mathbf{S t}(\mathcal{E})_{d}$ est la réunion des sous-ensembles constitués des transformations stellaires qui ont centre $\left(P_{1}, P_{2}\right)$ pour $P_{1}, P_{2} \in \mathbf{P}^{n}$; de plus, observer que les points génériques de ces sous-ensembles ne coïncident pas. On en déduit le résultat.

Remarque 3.1. En fait on peut montrer que si $\mathcal{E}_{1}, \mathcal{E}_{2} \subset \mathbf{C r}\left(\mathbf{P}^{n-1}\right)$ sont constitués par des éléments de degrés $r_{1}, r_{2}$ avec $r_{1} \neq r_{2}$, alors $\mathbf{S t}\left(\mathcal{E}_{1}\right)_{d} \cap \mathbf{S t}\left(\mathcal{E}_{2}\right)_{d}=\emptyset$, donc la proposition fournit une façon de calculer la dimension de l'ensemble des transformations stellaires de degré $d>2$.

Exemple 3.1. Dans le cas où $\emptyset \neq \mathcal{E} \subset P G L(n), \operatorname{dim} \mathbf{S t}(P G L(n))_{d}$ vaut

$$
n(n+2)+\frac{(n+d-3) !}{(d-2) !(n-1) !}\left[\frac{n^{2}+(4 d-3) n+2\left(2 d^{2}-4 d+1\right)}{d(d-1)}\right]
$$

et donc on a aussi

$$
\operatorname{dim} \mathbf{S t}(P G L(n))_{d} \approx 4 \prod_{l=0}^{d-3} \frac{l+n}{l+1}
$$

Comme cas particulier on en déduit une preuve du fait: $\operatorname{dim} \operatorname{Cr}\left(\mathbf{P}^{n}\right)=\infty$ si $n \geq 2$.

Exemple 3.2. Dans le cas des transformations stellaires de degré 2, on ne peut pas assurer l'unicité (générique) du sommet. Par exemple on sait (voir 2] ou encore [4]) qu'une transformation quadratique générique dans le plan est dans l'orbite de l'application $F_{2}$ de l'exemple 2.2 . 
Commentaires finals. Pour $n>2$ et $r=1$ on peut montrer l'unicité du centre sans aucune hypothèse de généricité: une telle transformation $F$ avec centre $\left(P_{1}, P_{2}\right)$ et $\left(P_{1}^{\prime}, P_{2}^{\prime}\right)$ doit vérifier qu'il existe $q_{1}, q_{1}^{\prime} \in A_{d-1}$ tel que

$$
\Lambda_{F} \supset \mathbf{P}\left(q_{1} V_{1}\right) \cup \mathbf{P}\left(q_{1}^{\prime} V_{1}^{\prime}\right),
$$

avec $V_{1}, V_{1}^{\prime} \subset A_{1}$ désignant les sous-espaces des formes s'annulant en $P_{1}$ et $P_{1}^{\prime}$ respectivement. Avec des arguments de divisibilité on montre qu'alors $q_{1}^{\prime} / q_{1} \in$ $k \backslash\{0\}$ et $P_{1}=P_{1}^{\prime}$; le même argument vaut pour $F^{-1}$.

\section{REFERENCES}

[1] L. Ein and N. Shepherd-Barron (1989): Some Special Cremona transformations. American Journal of Mathematics, 111, pages 783-800. MR 90j:14015

[2] L. Godeaux ( ): Géométrie algébrique I. Transformations birationnelles et géométrie hyperespatielle. Massons \& $C^{i e}$, Editeurs, Paris

[3] J. Harris (1992): Algebraic Geometry. Springer Verlag. MR 93j:14001

[4] H.P. Hudson (1927): Cremona transformation in Plane and Space. Cambridge at the University Press.

[5] B.Crauder and S.Katz (1989): Cremona Transformations with smooth irreducible fundamental locus. American Journal of Mathematics 111, 289-30\%. MR 90b:14012

[6] I. Pan (1999): Une remarque sur la génération du groupe de Cremona, Bol. Soc. Bras. Mat., Vol. 30, N.1, 95-98 MR 2000b:14015

[7] J.G. Semple and L. Roth (1949): Introduction to Algebraic Geometry. Oxford at the Clarendon Press, Amen House, London E.C.M. MR 11:535d

Instituto de Matemática-UfRGS, AV. Bento Gonçalves 9500, 91540-000 Porto AleGRE/RS, BRASIL

E-mail address: pan@mat.ufrgs.br 